\title{
In vitro biomechanical evaluation of internal fixation techniques on the canine lumbosacral junction
}

Peter Early, Peter Mente, Stacy L Dillard, Simon Roe

Few biomechanical studies have evaluated the effect of internal stabilization techniques after decompressive surgery on the stability of the canine lumbosacral junction. The purpose of this canine cadaver study is to evaluate the stability of the canine lumbosacral (LS) spine in flexion and extension following laminectomy and discectomy and then stabilization with each of the three techniques: pins and polymethylmethacrylate (P/PMMA), two dorsal locking plates (SOP) or bilateral transarticular facet screws (FACET). Using a cantilever biomechanical system, bending moments were applied to the LS and range of motion (ROM) was recorded via a rotational potentiometer. With $3 \mathrm{Nm}$, the ROM ( $n=4$ in each group) for P/PMMA, SOP and FACET were $1.92 \pm 0.96^{\circ}, 2.56 \pm 0.55^{\circ}$ and $3.18 \pm 1.14^{\circ}$, respectively. With moments up to $35 \mathrm{Nm}$, the P/PMMA specimens appeared stable. Sacroiliac motion in the SOP and FACET groups invalidated further comparisons. Each of the stabilization techniques (P/PMMA, SOP, and FACET) significantly decreased the range of motion in flexion and extension for low bending moments. 
2 In vitro biomechanical evaluation of internal fixation techniques on the canine lumbosacral junction.

4

5

6

7

8
P. Early ${ }^{a *}$, P. Mente ${ }^{b \dagger}$, S. Dillard ${ }^{c}$, S. Roe ${ }^{a}$

${ }^{a}$ North Carolina State University, College of Veterinary Medicine, 1052 William Moore Drive, Raleigh NC 27607 USA

${ }^{b}$ North Carolina State University, Joint Department of Biomedical Engineering at the University of North Carolina Chapel Hill and North Carolina State University, 4130 Engineering Building III, 911 Oval Drive, Raleigh NC 27695 USA

${ }^{c}$ Veterinary Neurology Center, 3051 Edinger Avenue, Tustin CA 92780 USA

$\dagger$ Deceased January 20, 2014

* Corresponding author. Tel.: +1 9195136692

E-mail address: pjearly@ncsu.edu (P. Early) 


\section{Abstract}

Few biomechanical studies have evaluated the effect of internal stabilization techniques

47 after decompressive surgery on the stability of the canine lumbosacral junction. The purpose of

48 this canine cadaver study is to evaluate the stability of the canine lumbosacral (LS) spine in

49 flexion and extension following laminectomy and discectomy and then stabilization with each of

50 the three techniques: pins and polymethylmethacrylate (P/PMMA), two dorsal locking plates

51 (SOP) or bilateral transarticular facet screws (FACET).

52 Using a cantilever biomechanical system, bending moments were applied to the LS and

53 range of motion $(\mathrm{ROM})$ was recorded via a rotational potentiometer. With $3 \mathrm{Nm}$, the $\mathrm{ROM}(\mathrm{n}=4$

54 in each group) for P/PMMA, SOP and FACET were $1.92 \pm 0.96^{\circ}, 2.56 \pm 0.55^{\circ}$ and $3.18 \pm 1.14^{\circ}$,

55 respectively. With moments up to $35 \mathrm{Nm}$, the P/PMMA specimens appeared stable. Sacroiliac

56 motion in the SOP and FACET groups invalidated further comparisons.

Each of the stabilization techniques (P/PMMA, SOP, and FACET) significantly

decreased the range of motion in flexion and extension for low bending moments.

60 Keywords: Lumbosacral; Biomechanics; Dog; PMMA; SOP; Transarticular Facet Screws

61

62

63

64

65

66

67 


\section{Introduction}

Degenerative Lumbosacral Stenosis (DLS) is a common cause of caudal lumbar pain, difficulty in sitting and difficulty rising in middle aged large breed dogs (Meij and Bergknut, 2010). DLS is commonly associated with Hansen type II disc degeneration, ligamentous hypertrophy, articular facet and joint capsule hypertrophy, spondylosis deformans, subluxation of the sacrum and lumbosacral instability. It is thought that increased motion at the lumbosacral junction is the most important contributor to the degenerative changes and progression of clinical signs in dogs (Meij and Bergknut, 2010).

Surgical management is recommended for patients with severe or recurrent pain that is not responsive to medical management or when neurologic deficits are present (Johnston and Tobias 2012a). Common surgical options for DLS include dorsal laminectomy alone or in combination with a partial discectomy, dorsal laminectomy combined with fixation and fusion or lateral foraminotomy (Meij et al., 2007, Hankin et al., 2012, Smolders et al., 2012bc). Two previous biomechanical studies have shown that a dorsal laminectomy with partial discectomy increases lumbosacral movement, which may lead to instability (Smolder et al., 2012b, Early et al., 2013). Some surgeons feel that dorsal stabilization is indicated to provide stability to the LS junction. If instability is present, then dorsal stabilization will limit the range of motion. Even in the absence of significant instability, if there is nerve impingement secondary to proliferation of tissue around the LS junction, rigid fixation may reduce the intensity of the fibrous response, thus relieving the pressure on the spinal nerves. An optimal configuration or system of fixation has yet to be determined. Two of the most widely used and clinically accepted fixation techniques are 1) positive profile threaded pins and polymethylmethacrylate (Weh and Kraus, 2007) and 2) bilateral transarticular facet screw stabilization (Hankin et al., 2012). The SOP TM 
91 locking plate system may also be suitable, enabling screws to be directed into the limited bone 92 available (Johnston and Tobias. 2012b).

93 Currently there remains inadequate cased-based evidence to support the use of surgical 94 intervention over conservative management for DLS (Jeffery et al, 2014). There seems to be 95 major controversy with regard to the type of surgery that may be chosen as well. Most 96 veterinarians would support surgical intervention in dogs with severe pain and fecal or urinary

97 incontinence. Of the common surgical options listed above, none is without limitations. Dorsal

98 laminectomy and discectomy may not alleviate compression of the L7 nerve in the foramen.

99 Foraminotomy alone does not allow for removal of protruding disc and ligamentous

100 compression within the spinal canal. Combining a dorsal laminectomy and foraminotomy may

101 increase the risk of articular facet fracture. Stabilization of the LS junction is performed when

102 the goal is to reduce the dynamic components of nerve compression within the vertebral canal,

103 or when it is the surgeon's opinion that the LS junction is unstable. Potential problems with the

104 LS stabilization techniques include poor implant placement (i.e. implants within the vertebral

105 canal) and inability to assure long term rigid fixation and bony fusion (Smolders et al., 2012c).

106 The purpose of this canine cadaver study was to evaluate the range of flexion and

107 extension and load to failure of the canine lumbosacral spine following stabilization with pins

108 and polymethylmethacrylate (P/PMMA), two dorsal locking plates (SOP) and bilateral

109 transarticular facet screws (FACET), after a dorsal laminectomy and partial discectomy.

\section{Materials and Methods}

111 The caudal lumbar spine (L6-7), sacrum and pelvis were harvested from 12 euthanized

112 skeletally mature non-chondrodystrophic dogs with weights ranging from 23.6-36.7 kg (median

$11330.4 \mathrm{~kg}$ ). Specimens were radiographed and no degenerative changes were noted. The pelvis 
114 was fixed in a resin mold (Bondo, Bondo Corporation, Atlanta, GA0, which was mounted to the

115 base of a testing machine (MTS, Canton, MA). An eyebolt screwed into the center of L6 was

116 attached to the actuator so that the spine segment could be flexed and extended. The applied

117 moment $(\mathrm{Nm})$ was calculated by multiplying the applied load $(\mathrm{N})$ by the distance from the LS

118 space to the actuator (m). Movement of L7 was monitored by a weighted rotational

119 potentiometer (P1411, Novotechnik, Southborough, MA) attached to the ventral aspect of the

120 vertebra. In a previously reported study, the specimens were conditioned at $\pm 1.5 \mathrm{Nm}$, at a rate of

121 two cycles per second and range of motion (ROM) were measured for $\pm 3 \mathrm{Nm}$ of bending (Early

122 et al., 2013). An L7-S1 dorsal laminectomy and partial discectomy was performed and the ROM

123 measured. The ROM of the intact specimens was $32.8 \pm 6.4^{\circ}$ and, after laminectomy and

124 discectomy, this increased to $40.2 \pm 5.6^{\circ}$ (Early et al., 2013).

125 Following the ROM analysis, one of the three fixation techniques (P/PMMA, SOP and

126 FACET) was applied to each of the specimens. There were 4 specimens per group. Implant

127 entry points for the three fixation techniques are identified in Figure 1 and radiographs depicting

128 each technique are given in Figure 2. The P/PMMA construct consisted of six positive profile

$1294.0 \mathrm{~mm}(5 / 32 ”)$ external fixation half-pins (Interface TM, Imex Veterinary, Inc., Longveiw, TX).

130 Two pins were placed into the pedicle of L7, two pins into the sacrum and two pins into the

131 ilium. Predrilling for pins was not performed. PMMA was applied, incorporating all of the pins

132 (Fossum 1997). The PMMA was contoured and in close contact with the bone surface of L7

133 and sacrum. The PMMA was allowed to harden for a minimum of 1 hour before testing.

134 The SOP fixation consisted of two 5-hole $3.5 \mathrm{~mm}$ locking plates (SOPTM, Orthomed Ltd.,

135 West Yorkshire, UK) that were positioned parallel on either side dorsolaterally and secured to

136 the pedicle of L7 and sacrum with two $3.5 \mathrm{~mm}$ cortical screws (Depuy Synthes Vet, West 
137 Chester, PA) in each plate. All of the screws for the SOP construct were placed in the most

138 cranial pearl (hole 1) skipping the second pearl and then placing the second screw in the third 139 pearl (hole 3 ).

140 The FACET fixation consisted of two $3.5 \mathrm{~mm}$ cortical screws oriented from the dorsal

141 articular processes of L7, into the sacrum using a positional technique (Sharp and Wheeler. 142 2005).

143 After each fixation technique was applied, the specimen was preconditioned at $\pm 1.5 \mathrm{Nm}$

144 for 5 cycles, then loaded at $\pm 3 \mathrm{Nm}$ for 5 cycles to measure ROM. Subsequently, the stabilized

145 specimens were subject to an incrementally increasing load, starting at $\pm 2.5 \mathrm{Nm}$ and increasing

146 by $2.5 \mathrm{Nm}$ after each set of 5 loading cycles, until testing was concluded. Testing was

147 concluded if: 1) motion of L7 was greater than $10^{\circ}$ in flexion or extension, 2) implant failure or

148 bony fracture occurred; or 3) a bending moment of $35 \mathrm{Nm}$ was applied (Smith et al. 2004).

149 After all ROM testing was complete, lateral and dorsoventral radiographs were made of all 150 specimens and the failure mechanism evaluated on these and on the specimens.

151 The ROM with $\pm 3.5 \mathrm{Nm}$ applied moment was compared between the stabilized

152 specimens and the intact and decompressed data available from a previous study (Early et al,

153 2013). If differences were identified using ANOVA, individual comparisons were made using

154 the least squares means test, and an overall P value of 0.05 to determine significance (SAS

155 v9.1.3 Service pack 4, SAS Institute Inc., Cary, NC). Because of issues identified during the

156 incremental load to failure study, statistical comparison of load to failure data was not 157 performed.

158 Results 
159 The ROM with $\pm 3.5 \mathrm{Nm}$ for the P/PMMA, SOP and FACET techniques were $1.92 \pm 0.96^{\circ}$,

$1602.56 \pm 0.55^{\circ}$, and $3.18 \pm 1.14^{\circ}$ respectively, Figure 3. After each fixation technique was applied

161 the ROM of the stabilized specimens was significantly decreased $(p<0.001)$ compared to ROM

162 after dorsal laminectomy and discectomy (mean of all specimens for all three groups $=40.2 \pm 5.6^{\circ}$

163 (Early et al., 2013). One of the FACET specimens failed because of fracture around the screw

164 with $14.1 \mathrm{Nm}$ applied while in extension. One of the SOP specimens failed by loosening of the

165 screws in L7 with $12.7 \mathrm{Nm}$ applied while in extension. The other three specimens in each of the

166 FACET and SOP groups failed because L7 motion was greater than $10^{\circ}$, though most of that

167 motion originated at the SI joints. There was no failure of the fixation noted on gross inspection,

168 or on radiographs. In the P/PMMA group, testing was stopped at $35 \mathrm{Nm}$ of bending for three

169 specimens, with no implant failure noted on gross inspection, or on radiographs. In the other

170 P/PMMA specimen, the eyebolt fractured through L6 when a $25 \mathrm{Nm}$ moment was applied.

\section{Discussion}

172 This study demonstrates that the LS region had much less range of motion after

173 stabilization with each fixation technique, but, because the P/PMMA technique bridges the SI

174 joint, and the FACET and SOP techniques did not, the specimens moved very differently during

175 testing at higher bending moments. For this reason we felt that it was not appropriate to make

176 direct comparisons of failure using the mechanical data.

177 Visually and mechanically, the P/PMMA technique appeared to provide good stability.

178 Three of four specimens resisted the highest applied moment with no evidence of failure. As the

179 flexion and extension moment was increased on the FACET and SOP specimens, there was

180 increasing motion of the SI joint. As this allowed L7 to move relative to the pelvis, this motion 
181 was included in the data. Movement of L7 relative to S1 could not be separated from the SI 182 motion.

183 Visually, no motion was observed in the SOP specimens, except in one, where one screw

184 loosened in L7. This suggests that, in this configuration and testing mode, the screw-bone

185 interface was the weaker element. This may be due to slightly incorrect placement of this

186 particular screw, or to the fact that there were only 2 screws in each vertebrae. There is a very

187 narrow region into which the screw is inserted in L7 to optimize its purchase, while not

188 damaging adjacent structures (Smolders et al., 2012c). The recently developed SOPTM Locking

189 Plate System combines the advantages of a fixed angle stabilization system, like the P/PMMA,

190 with lower bulk. The SOPTM plate can be contoured so that the locked screws are directed into

191 the limited available bone stock (Johnston and Tobias 2012b). When using the SOP ${ }^{\mathrm{TM}}$ locking

192 plate system in the lumbar and sacral vertebrae, the following guidelines have been

193 recommended recently - use SOP locking plates bilaterally, twist and contour the SOP caudally

194 to engage the iliac shaft, recommend 4 screws but a minimum of 3 screws in each vertebral

195 body, use the longest possible cortical screws to engage maximum amount of vertebral bone and

196 have the SOP plate as close to the bone as possible while avoiding damage to emerging nerve

197 roots (Orthomed product information brochure). The configuration used in this study was

198 selected before the above recommendations were available. The 2-screw configuration was

199 chosen to mimic the pedicle rod and screw stabilization technique commonly used in humans

200 and by some surgeons in dogs (Smolders et al., 2012c).

201 In the FACET specimens, slight motion was apparent between the facets. Fracture of the

202 facet because it is weakened by the screw, as occurred in one specimen, is a known potential

203 complication of this technique (Sharp \& Wheeler, 2005, Hankin et al., 2012) 
Previous in vitro cadaver studies have evaluated the biomechanical effects of

stabilization after concurrent dorsal laminectomy and partial discectomy on the lumbosacral

206 junction in the dog have yielded similar results (Meij et al., 2007, Smolders et al., 2012a). In the

207 Meij et al. 2007 study, a pedicle screw and rod fixation significantly stabilized the lumbosacral

208 spine by decreasing the ROM from $29.1 \pm 5.60^{\circ}$ to $11.7 \pm 3.30^{\circ}$. In the Smolders et al. 2012a

209 study, a nucleus pulposus prosthesis effectively decreased the ROM of the lumbosacral spine by

$2108.8 \%$. These studies evaluated the lumbosacral spine segments using 4-point bending. In this

211 study, a cantilever system was used as it was easier to instrument and load the spinal segment.

212 While the applied moment varies over the length of the specimen in cantilever bending, the

213 moment applied to the LS articulation is easily calculated.

214 This study was intended to evaluate clinically accepted techniques for stabilization of the

215 LS junction. Several distinctions should be noted as these potentially alter and give significant

216 advantages to the various biomechanics of each construct. The P/PMMA fixation has the

217 potential advantage of six screws and points of fixation, while the SOP and FACET have four

218 and two points of fixation respectively. The P/PMMA constructs used $4.0 \mathrm{~mm}$ positive profile

219 pins with a $3.2 \mathrm{~mm}$ shaft, while the SOP and FACET constructs used $3.5 \mathrm{~mm}$ cortical screws,

220 with a $2.4 \mathrm{~mm}$ shaft. The P/PMMA construct was thicker, bulkier and in more intimate contact

221 with the L7 and sacral vertebrae providing a buttress stabilization, which likely contributed to

222 the more stable appearance of this group.

An interesting finding of this study was that the motion at the sacroiliac (SI) joint was not constant between stabilization techniques. The P/PMMA technique stabilized the SI joint such that no motion was appreciated at that articulation. The SI joint was not stabilized in the SOP

226 and FACET specimens. It is unknown if preserving SI motion may have a clinical advantage. In 
227 the recommendations for SOP placement described above, bridging the SI joint is advised, so SI

228 motion would be lost if this was performed. Anchoring implants to the ilium is suggested

229 because of the historically poor screw purchase achieved in the sacrum alone.

230 Cantilever bending does typically result in a higher bending moment at the point of

231 fixation of the specimen to the rigid stand compared to at the tip where the load is applied. In

232 contrast, an even bending moment is applied with a 4-point fixture. The cantilever model was

233 selected because it appeared to replicate the loads that would be applied to the LS region when

234 the hind limbs were in stance phase and the load of the front half of the body was acting on the

235 lumbar spine. Because the ilial shafts were potted right up to the sacrum, the highest bending

236 moments would have been present on the SI joints and the LS joint. Of course, all models of

237 spine motion are gross simplifications since they disregard the very important contribution of the 238 active stabilizers of the system.

239 The primary limitation of this study was that, because SI motion was much greater than

240 expected, we were not able to compare the failure properties of the three different stabilization

241 techniques. Another limitation of the study design was that the ability of the fixation methods to

242 resist lateral bending and axial rotational forces was not evaluated.

\section{Conclusion}

244 This cadaver study demonstrated that stabilization of the lumbosacral junction by all

245 three of the applied fixation techniques leads to decreased motion in flexion and extension. It is

246 unknown whether the stabilization techniques used in combination with the dorsal laminectomy

247 and partial discectomy procedures will provide sufficient stability for healing in clinical cases.

248 Acknowledgements 
We thank Kristal Wilson for her assistance in acquiring data in the bioengineering

250

251

252

253

254

255

256

257

258

259

260

261

262

263

264

265

266

267

268

269

270

271

272

273

274

275

276

277

278

279

280

281

282

283

284

285

286

287

288

289

290

291

laboratory and Robert Thatcher for his assistance with the figures.

\section{References}

Benninger, M., Seiler, G., Robinson, L. Ferguson, S., Bonél, H., Busato, A., Lang, J., 2004.

Three-dimensional motion pattern of the caudal lumbar and lumbosacral portions of the vertebral column of dogs. American Journal of Veterinary Research 65:544-551.

Early, P., Mente, P., Dillard, S., Roe, S., 2013. In vitro biomechanical comparison of the canine lumbosacral vertebrae before and after dorsal laminectomy and partial discectomy. The Veterinary Journal 196:533-535.

Fossum, T.W., 1997. Small Animal Surgery: Surgery of the Lumbosacral Spine, First Ed. Mosby, St. Louis, MO, USA, pp. 1131-1149.

Hankin, E., Jerram, R., Walker, A., King, M., Warman, C. 2012. Transarticular Facet Screw Stabilization and Dorsal Laminectomy in 26 Dogs with Degenerative Lumbosacral Stenosis with Instability. Veterinary Surgery 41:611-619.

Jeffery N., Barker A., Harcourt-Brown T.2014. What progress has been made in the understanding and treatment of degenerative lumbosacral stenosis in dogs during the past 30 years? The Veterinary Journal 201, 9-14.

Johnston, S.A. and Tobias, K.M., 2012a. Veterinary Surgery: Lumbosacral Spine, Second 180 Ed. Elsevier Saunders, St. Louis, MO, USA, pp. 476-486.

Johnston, S.A. and Tobias, K.M., 2012b. Veterinary Surgery: Spinal Fractures and Luxations, Second Ed. Elsevier Saunders, St. Louis, MO, USA, pp. 496-497.

Meij, B., Suwankong, N., van der Veen, A., Hazewinkel, H., 2007. Biomechanical flexionextension forces in normal canine lumbosacral cadaver specimens before and after dorsal laminectomy-discectomy and pedicle screw-rod fixation. Veterinary Surgery 36:742-751.

Meij, B.P., Bergknut, N., 2010. Degenerative lumbosacral stenosis in dogs.

The Veterinary Clinics of North America: Small Animal Practice 40:983-1009.

Sharp, N. J. and Wheeler S.J. 2005. Lumbosacral Disease in Small Animal Spinal Disorders: Diagnosis and Surgery. Mosby, St Louis, MO, USA, pp 181-209.

Smith, M.E.H., Bebchuk, T.N., Shmon, C.L, Watson, L.G., Steinmetz, H., 2004. An in vitro biomechanical study of the effects of the surgical modification upon the canine lumbosacral spine. Veterinary and Comparative Orthopaedic and Traumalogy. 1:17-24 
292 Smolders, L., Bergknut, K., Kingma, I., van der Veen, A., Smit, T., Koole, L., Hazewinkel, H., 293 Meij B., 2012a. Biomechanical evaluation of a novel nucleus pulposus prosthesis in canine

294 cadaveric spines. The Veterinary Journal 192:199-205

295

296

297

298

299

300

301

302

303

304

305

306

307

308

309

310

311

312

313

314

315

316

Smolders, L.A., Kingma, I., Bergknut, N., van der Veen, A., Dhert, W.J., Hazewinkel, H., van Dieen, J.H., Meij, B., 2012b. Biomechanical assessment of the effects of decompressive surgery in non-chondrodystrophic and chondrodystrophic canine multisegmented lumbar spines.

European Spine Journal 21:1692-1699.

Smolders, L.A., Voorhout, G., van der Veen, R., Bergknut, N., Grinwis, G.C., Hazewinkel, H., Meij, B., 2012c. Pedicle Screw-Rod Fixation of the Canine Lumbosacral Junction. Veterinary Surgery 41:720-732.

Weh, J., Kraus, K. 2007. Use of a Four Pin and Methylmethacrylate Fixation in L7 and the Iliac Body to Stabilize Lumbosacral Fracture-Luxations: A Clinical And Anatomic Study. Veterinary Surgery 36:775-782.

Orthomed product information brochure. Author and publish date unknown. Standard Operating Procedures for SOP Fixation of Fractures, Orthomed (UK), Ltd, West Yorkshire, United Kingdom. Downloaded from:

http://www.orthomed.co.uk/download/?f=20100513115741 sopfixationoffractures web.pdf , May, 2015. 
Figure 1 (on next page)

Implants points of entry into L7, Sacrum and llium.

Dorsal view of the skeletal structures of the canine lumbosacral junction, showing the points of entry of the implants into L7, Sacrum and llium. The external fixation pins (for the P/PMMA) entry points are denoted with open dark gray circles with cross marks in the middle. The SOP locking plate entry points are denoted by solid black circles and the bilateral transarticular facet screws entry points and directions are denoted by black arrows. 
Figure 2 (on next page)

Postoperative radiographs of the three stabilization techniques.

Postoperative radiographs, lateral and dorsoventral, of the three stabilization techniques. (A)

External fixation pins and PMAA, (B) SOP ${ }^{\mathrm{TM}}$ Locking Plate System and (C) Bilateral transarticular facet screws. 


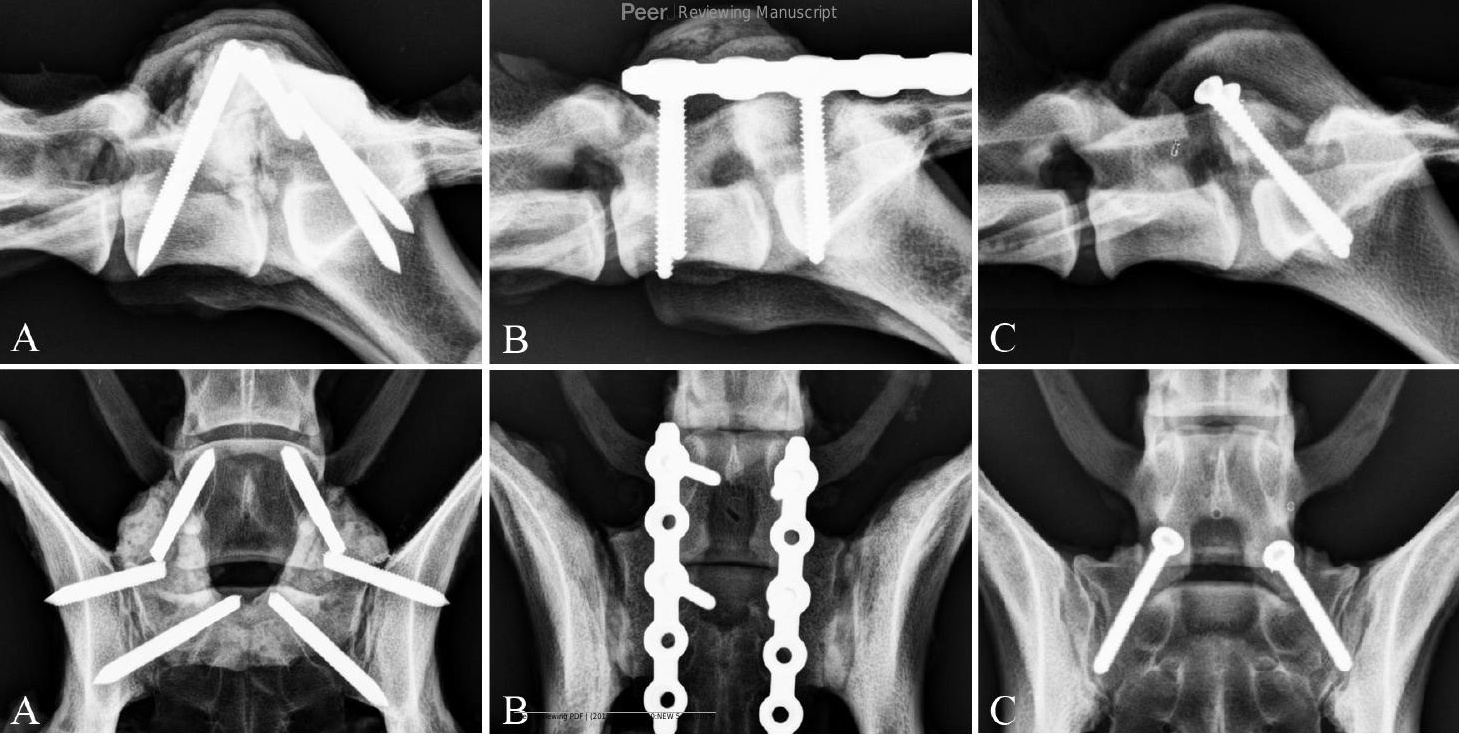


Figure 3 (on next page)

Typical load-deflection curve in a canine cadaver lumbosacral spine during cyclic loading of spines after dorsal laminectomy and partial discectomy and each stabilization technique.

Typical load-deflection curve in a canine cadaver lumbosacral spine during cyclic loading (flexion and extension) of spines after dorsal laminectomy and partial discectomy (DL) and each stabilization technique (SOP - black solid, FACET - light grey solid and P/PMMA - dark grey dashed). Range of motion (ROM) was the L7 angulation change between flexion and extension with $3 \mathrm{Nm}$ of bending moment applied. 
PeerJ Reviewing Manuscript

Flexion / Extension

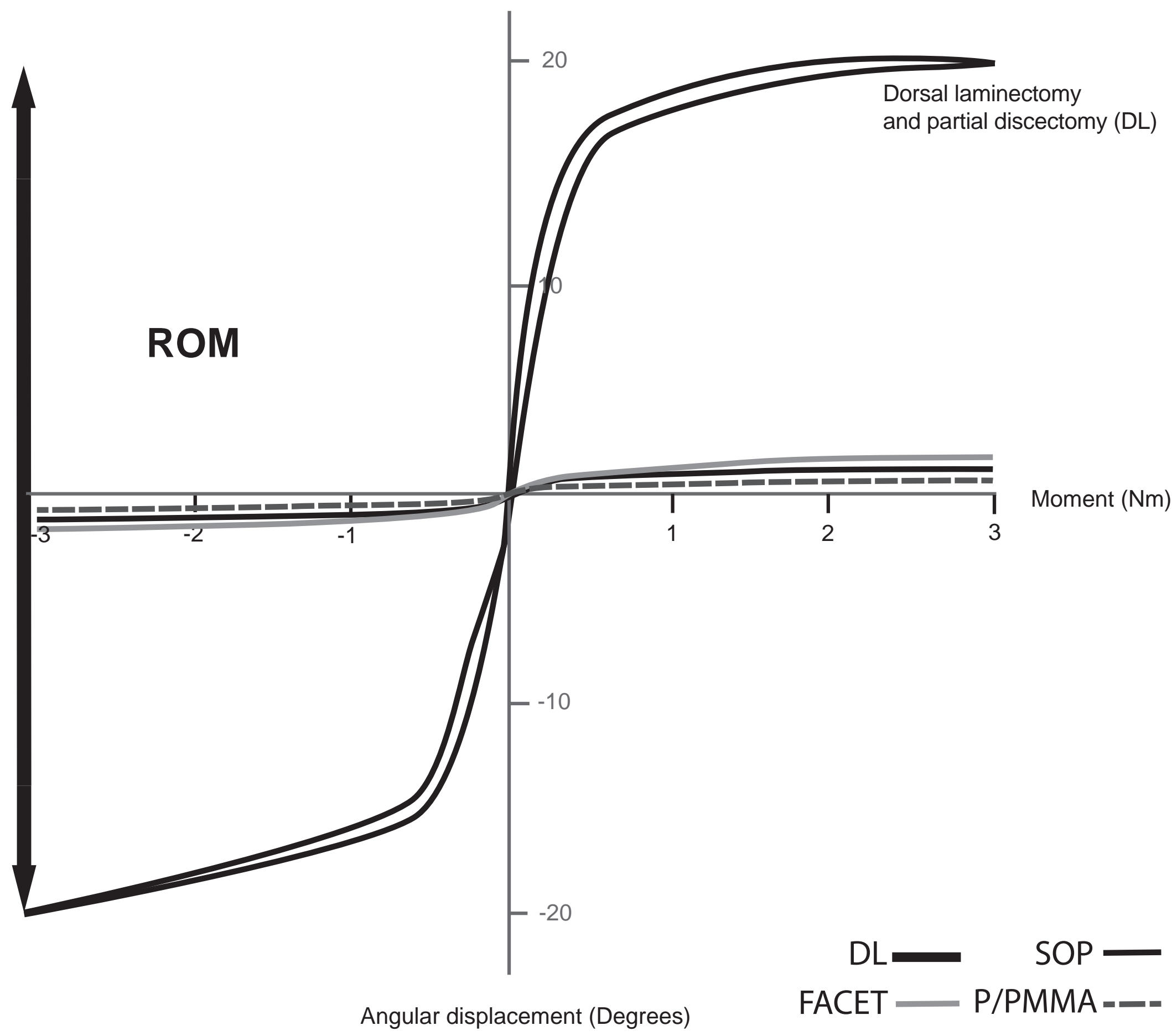

